\title{
Effects of Segmented Contractions of Gaussian Basis Sets on Dirac-Fock Energies
}

\author{
F. E. Jorge \\ Departamento de Fúsica \\ Universidade Federal do Espirito Santo \\ 29060-900, Vitória, ES, Brazil \\ Received 14 June, 1999
}

\begin{abstract}
Dirac-Fock calculations have been performed using a segmented contraction of Gaussian basis sets entirely generated from the Dirac-Fock Hamiltonian. Both Dirac-Fock-Coulomb and Dirac-FockBreit self-consistent field calculations have been performed for $\operatorname{Ar}(Z=18)$, Ge $(Z=32)$, Sn $(Z=50)$, $\mathrm{Xe}(\mathrm{Z}=54)$ and $\mathrm{Hg}(\mathrm{Z}=80)$. It is found that contractions of large exponent bases of $\mathrm{s}$ and $\mathrm{p}$ symmetry species have little effect on the total and orbital energies for light atoms ( $\mathrm{Ar}$ and $\mathrm{Ge}$ ) and a large effect for intermediate ( $\mathrm{Sn}$ and $\mathrm{Xe}$ ) and heavy ( $\mathrm{Hg}$ ) atoms.
\end{abstract}

\section{Introduction}

Recently, we have developed the closed-shell generator coordinate Dirac-Fock (GCDF) method [1,2] and applied it to perform Dirac-Fock-Coulomb (DFC) and Dirac- Fock-Breit (DFB) calculations on relativistic closed-shell atoms [1-5]. Here we would like to call attention for the fact that the GCDF method represents an algorithm capable of generating Gaussian-type functions (GTF) exponents directly from the relativistic environment [a Dirac-Fock (DF) code], whereas the usual procedure in previous relativistic calculations was to employ GTF exponents obtained from the nonrelativistic environment [a Hartree-Fock (HF) code], by optimization or another technique, exactly as first performed by Matsuoka and Huzinaga [6].

Thus, due to the recent progress in the DF methods, the problem of how to contract atomic Gaussian basis sets for use in these calculations becomes relevant. The general and the segmented contraction schemes [7-11] have been used in relativistic calculations. Matsuoka [7] and Ishikawa et al. [8-10] have described some segmented schemes to contract Gaussian basis sets they employed in relativistic atomic calculations In these papers, Matsuoka and Ishikawa worked with Gaussian basis sets that originally were generated to perform nonrelativistic calculations (with a $\mathrm{HF}$ code).

In this work, we report a DFC and DFB selfconsistent field (SCF) calculations for Ar, Ge, Sn, Xe and $\mathrm{Hg}$ using a segmented contraction methodology for Gaussian basis sets developed recently by Jorge and da Silva [12], in order to ascertain how contraction of the basis set affects DF energy results.

\section{Results and discussion}

To perform the DFC and DFB calculations for Ar, Ge, $\mathrm{Sn}$, Xe and $\mathrm{Hg}$, we have used our accurate universal Gaussian basis sets (UGBSs) [1,3], developed with the characteristic of being entirely generated from the DF Hamiltonian. This point is very important as all previous studies on segmented contraction of GTF for relativistic calculations were done with GTF obtained from the HF Hamiltonian [7-11]. Therefore in this work, the effects of a segmented contraction on DF energies are studied with Gaussian basis sets entirely generated from the DF Hamiltonian. The finite nucleus model of uniform proton-charge [13] with nuclear radius equal to $R=2.2677 \times 10^{-5} A^{1 / 3}$ ( $\mathrm{A}$ is the atomic mass number) was used in our calculations. The speed of light was taken to be 137.0370 a.u. while the nonrelativistic limit was evaluated with $c=10^{4}$ a.u.. Beside this, we recall that the radial small component functions are generated from the radial large component functions by using the restricted kinetic balance condition [ 13,14].

The procedure used by us to generate the segmented contraction schemes for each atom studied is the same used recently by Jorge and da Silva [12]. First for each atom under study, we tested the best nonrelativistic contraction scheme (the scheme that provides the best HF energy). Then, verifying that these contraction schemes provided a very poor DFC energy for the heavier atoms $\mathrm{Sn}$. Xe and $\mathrm{Hg}$ (although providing 
a reasonable one for the light atoms Ar and $\mathrm{Ge}$ ), we decided to change the strategy and start searching for the best relativistic contraction scheme. Here, for each atom ( $\mathrm{Ar}, \mathrm{Ge}, \mathrm{Sn}$, Xe and $\mathrm{Hg}$ ), we calculated the contraction coefficients for nonrelativistic orbitals and used them to evaluate the DFC energy. This procedure was repeated until finding a contraction scheme that provided a satisfactory DFC energy. At the end, we used the same set of exponents (and of the contraction coefficients, when is the case) to perform the corresponding DFB calculations. Here, we would like to call attention to the fact that all contraction schemes showed in Table I (for $\mathrm{Ar}, \mathrm{Ge}, \mathrm{Sn}, \mathrm{Xe}$ and $\mathrm{Hg}$ ) are relativistic contraction schemes.

Table I shows the relativistic segmented contraction schemes adopted for the Ar, Ge, Sn, Xe and Hg. The
UGBSs used here can be found elsewhere $[1,3]$. and they consist of $32 \mathrm{~s} 24 \mathrm{p}$ for Ar [3], 32s30p20d for Ge, Sn and Xe [1], and 32s30p20d14f for Hg [1]. From Table I, one can clearly see that for the light atoms (Ar and Ge) the core region can be contracted without restrictions, i.e., the contraction of large exponents (exponents that describe the core region) for the $\mathrm{s}$ and $\mathrm{p}$ symmetries has little effect on the total DFC energy, while the valence region (small exponents) can not be contracted strongly, as they have a large effect on the total DFC energy. By other hand, the valence region exponents only allow a very slight contraction. This behavior for the valence region is also true not only for the $s$ and $p$ symmetries of intermediate and heavy atoms, but also for the $\mathrm{d}$ and $\mathrm{f}$ symmetries of all atoms studied.

Table I. Segmented contraction schemes

\begin{tabular}{|c|c|c|c|}
\hline Atom & $\begin{array}{l}\text { Uncontracted } \\
\text { basis set size }\end{array}$ & Contraction schemes for $\mathrm{s} / \mathrm{p} / \mathrm{d} / \mathrm{f}$ symmetries & $\begin{array}{l}\text { Contracted } \\
\text { basis set size }\end{array}$ \\
\hline $\mathrm{Ar}$ & $32 \mathrm{~s} 24 \mathrm{p}^{\mathrm{a}}$ & $14,3,1^{12}, 3 / 13,2,1^{4}, 2^{2}, 1$ & {$[15 \mathrm{~s} 9 \mathrm{p}]$} \\
\hline $\mathrm{Ge}$ & $32 \mathrm{~s} 30 \mathrm{p} 20 \mathrm{~d}^{\mathrm{b}}$ & $10,2^{2}, 3,1^{9} 3,1^{3} / 11,2^{5}, 1^{3}, 2,1^{4} / 9,2,3^{3}$ & {$[17 \mathrm{~s} 14 \mathrm{p} 5 \mathrm{~d}]$} \\
\hline $\mathrm{Sn}$ & $32 \mathrm{~s} 30 \mathrm{p} 20 \mathrm{~d}^{\mathrm{b}}$ & $3^{2}, 2^{2}, 3,2,1^{11}, 3^{2} / 2,3,2^{2}, 3,2,1^{2}, 2,1^{5}, 2,1^{5} / 9,2,1^{3}, 3^{2}$ & {$[19 \mathrm{~s} 20 \mathrm{p} 7 \mathrm{~d}]$} \\
\hline $\mathrm{Xe}$ & $32 \mathrm{~s} 30 \mathrm{p} 20 \mathrm{~d}^{\mathrm{b}}$ & $3^{2}, 2^{2}, 3,2,1^{11}, 3^{2} / 2,3,2^{2}, 3,2,1^{2}, 2,1^{7}, 3,2 / 9,2,1^{3}, 3^{2}$ & {$[19 \mathrm{~s} 18 \mathrm{p} 7 \mathrm{~d}]$} \\
\hline $\mathrm{Hg}$ & $32 \mathrm{~s} 30 \mathrm{p} 20 \mathrm{~d} 14 \mathrm{f}^{\mathrm{b}}$ & $3^{2}, 2^{2}, 3,2,1^{11}, 3,3, / 2,3,2,1^{2}, 2,1^{3}, 2^{2}, 1^{6}, 3^{2} / 9,2,1^{5}, 2^{2} / 5,4,2,3$ & {$[19 \mathrm{~s} 19 \mathrm{p} 9 \mathrm{~d} 4 \mathrm{f}]$} \\
\hline
\end{tabular}

The relativistic contraction scheme for the core region changes completely when one tries to contract a GTF for heavier atoms (from Sn on). From Table I one can see that the segmented contraction scheme for the $\mathrm{s}$ and p symmetries for the core region of $\mathrm{Sn}$, $\mathrm{Xe}$ and $\mathrm{Hg}$ is not the same as of Ar and Ge, since we can not contract the large exponents of the $\mathrm{s}$ and $\mathrm{p}$ symmetries so easily as we do for Ar and Ge. In fact, the flexibility of contracting the large exponents for $\mathrm{s}$ and $\mathrm{p}$ symmetries decreases when the atomic number $\mathrm{Z}$ increases $(\mathrm{Z}=18$ for $\mathrm{Ar}, \mathrm{Z}=32$ for Ge, $\mathrm{Z}=50$ for $\mathrm{Sn}, \mathrm{Z}=54$ for $\mathrm{Xe}$, and $\mathrm{Z}=80$ for $\mathrm{Hg}$ ). For $\mathrm{d}$ and $\mathrm{f}$ symmetries this restriction is not so imperative, as we can see from Table 1, where the contraction of the large exponents of the $d$ and $f$ symmetries without severe restrictions is evident.

In Tables II, III and IV, $E_{\mathrm{DFC}}$ and $E_{\mathrm{DFB}}$ denote the DFC and DFB energies, respectively. $E_{\mathrm{NR}}$ denotes the energy taken at the nonrelativistic limit. Also shown are the energy differences between the DFC and nonrelativistic $\mathrm{SCF}$ energies $\Delta E_{\mathrm{NR}-\mathrm{DFC}}$, i.e., the lowering of the total HF energy due to relativistic effects. Beside this, it is shown the variational Breit interaction energies, $E_{B}$, which is the level shift in the total SCF energy due to the inclusion of the Breit term in the 
SCF process, and is computed as the ditference, $E_{\mathrm{DFB}}$ - EDFC.

Table II contains the DFC and DFB anergies for Ar $(Z=18)$, Ge $(Z=32)$, Sn $(Z=50)$, $X e(Z=54)$ and $\mathrm{Hg}(\mathrm{Z}=80)$ computed with the UGBSs $[1,3]$ in uncontracted (see the third column) and contracted (see the fourth column) form. For Ar, Ge, Sn, Xe and $\mathrm{Hg}$ the difference between the DFC energies obtained with the relativistic contracted and uncontracted basis sets are $0.000147,0.00665,0.07103,0.06655$ and 0.9859 hartree, respectively. As the DFC energies obtained with the uncontracted basis sets are approximately equal to or lightly lower than the corresponding values obtained with the numerical DF calculations [15] (see fifth column), the differences between the DFC energies obtained with our relativistic contracted basis sets and the numerical DF calculations are approximately equal to the differences shown above. For Ar, the Breit interaction energies $E_{B}$ calculated with our relativistic uncontracted and with the contracted basis sets are the same. For Ge, Sn, and Xe the diferences in $E_{B}$ obtained from these two basis sets are of the order of $10^{-4}$ hartree, and for $\mathrm{Hg}$ this difference increases to $10^{-2}$ hartree. From Table II, we can also see that the differences between the relativistic energy lowering, $\Delta E_{\mathrm{NR}-\mathrm{DFC}}$, obtained with our uncontracted and with the contracted basis sets for the light ( $\mathrm{Ar}$ and $\mathrm{Ge}$ ), intermediate ( $\mathrm{Sn}$ and $\mathrm{Xe})$ and heavy $(\mathrm{Hg})$ atoms are lower than $3.6 \times 10^{-3}$, $4.6 \times 10^{-2}$, and $8.7 \times 10^{-1}$ hartree, respectively.

Tables III and IV display the orbital and the total DF energies of Ge and Sn, respectively, computed by employing the uncontracted and contracted basis sets given by us and in Aerts's thesis [18]. From these Tables, we can see that the orbitals energies obtained using the relativistic uncontracted basis set [1] (see the second columns) are in general more stable than those obtained with our relativistic contracted basis sets (see the third columns). On the other hand, the results reported by Aerts and Nieuwpoort [18] show that, for both Ge and Sn, their contraction schemes (see the seventh columns) causes the $1 \mathrm{~s}_{1 / 2}$ energy to shift downward by a significant amount [18]. This shift in the orbital energy, in turn, forces the total DFC energy to shift well bellow that obtained with the nonrelativistic uncontracted basis set causing variational failure. The Yshikawa et. al. results $[8,19]$ (see the fourth and fifth columns from Tables III and IV) also are in contradiction to the results reported by Aerts and Nieuwpoort. For Ge and Sn, our DF calculations employing relativistic contracted basis sets indicate that the $1 \mathrm{~s}_{1 / 2}$ energies shift upward by 0.002 and 0.003 hartree, respectively, whereas a significant amount of downward shift $(\approx 0.2$ hartree for $\mathrm{Ge}$ and $\approx 4.2$ hartree for $\mathrm{Sn}$ ) is evident in the DF calculation by Aerts and Nieuwpoort [18].

From Tables III and IV we can also see that in nonrelativistic calculations, the total HF energies for Ge and Sn computed by using the contracted basis sets of the three approaches are always higher than those obtained by using the uncontracted basis sets, because the Schroedinger Hamiltonian is bounded from below. In the DF calculations, the variational failure may readily be understood $[11,20]$ as a consequence of the unbounded nature of the relativistic Hamiltonian. This is especially important for the innershell $1 \mathrm{~s}_{1 / 2}$ and $2 \mathrm{p}_{1 / 2}$ orbitals if a contracted basis set is employed in the DF calculations which do not satisfy the restricted kinetic balance condition $[13,14]$ between the radial large and radial small component functions.

\section{Conclusions}

The DF energy values reported in this work for Ar $(Z=18)$, Ge $(Z=32)$, Sn $(Z=50)$, Xe $(Z=54)$ and $\mathrm{Hg}$ $(\mathrm{Z}=80)$ show that heavy contractions of large exponent bases of $\mathrm{s}$ and $\mathrm{p}$ symmetries have little effect on the total and orbital energies for light atoms, but have large effect for intermediate and heavy atoms. The large Gaussian function exponents of the $\mathrm{d}$ and $\mathrm{f}$ symmetries for light, intermediate and heavy atoms allow a high degree of contraction. Besides this, our results show that we are not allowed to perform a strong contraction with the small Gaussian function exponents for $\mathrm{s}, \mathrm{p}, \mathrm{d}$ and $\mathrm{f}$ symmetries in any case (light, intermediate and heavy atoms).

\section{Acknowledgments}

The author is very grateful to Dr. L. T. Peixoto at Universidade Federal do Espírito Santo for valuable discussions and to Professor Y. Ishikawa for the DF SCF program. I would like to gratefully acknowledge the financial support by CNPq (Brazilian Agency). 
Table II. Energy values (in hartree) of the DFC and DFB calculations obtained by using uncontracted and contracted basis sets and numerical calculations

\begin{tabular}{|c|c|c|c|c|}
\hline & $\begin{array}{c}\text { Atomic } \\
\text { mass }\end{array}$ & $\begin{array}{c}\text { Uncontracted } \\
\text { basis set }\end{array}$ & $\begin{array}{c}\text { Contracted basis } \\
\text { set (this work) }\end{array}$ & $\begin{array}{l}\text { Numerical } \\
\text { calculations }\end{array}$ \\
\hline Ar & 39.948 & $32 \mathrm{~s} 24 \mathrm{p}^{\mathrm{a}}$ & {$[15 s 9 p]$} & \\
\hline $\mathrm{E}_{\mathrm{DFC}}$ & & -528.683823 & -528.683676 & $-528.683840^{\circ}$ \\
\hline $\mathrm{E}_{\mathrm{DFB}}$ & & -528.551500 & -528.551353 & \\
\hline $\mathrm{E}_{\mathrm{NR}}$ & & -526.817331 & -526.817318 & $-526.817513^{d}$ \\
\hline$\Delta \mathrm{E}_{\mathrm{NR}-\mathrm{DFC}}$ & & 1.866492 & 1.866358 & 1.866327 \\
\hline $\mathrm{E}_{\mathrm{B}}$ & & 0.132323 & 0.132323 & \\
\hline $\mathrm{Ge}$ & 72.59 & $32 \mathrm{~s} 30 \mathrm{p} 20 \mathrm{~d}^{\mathrm{b}}$ & {$[17 \mathrm{~s} 14 \mathrm{p} 5 \mathrm{~d}]$} & \\
\hline $\mathrm{E}_{\mathrm{DFC}}$ & & -2097.46672 & -2097.46007 & $-2097.46675^{c}$ \\
\hline $\mathrm{E}_{\mathrm{DFB}}$ & & -2096.51666 & -2096.51011 & \\
\hline $\mathrm{E}_{\mathrm{NR}}$ & & -2075.32373 & -2075.31357 & $-2075.35973^{d}$ \\
\hline$\Delta \mathrm{E}_{\mathrm{NR}-\mathrm{DFC}}$ & & 22.14299 & 22.14650 & 22.10702 \\
\hline $\mathrm{E}_{\mathrm{B}}$ & & 0.95006 & 0.94996 & \\
\hline $\mathrm{Sn}$ & 118.69 & $32 \mathrm{~s} 30 \mathrm{p} 20 \mathrm{~d}^{\mathrm{b}}$ & {$[19 \mathrm{~s} 20 \mathrm{p} 7 \mathrm{~d}]$} & \\
\hline $\mathrm{E}_{\mathrm{DFC}}$ & & -6176.14098 & -6176.06995 & $-6176.14105^{c}$ \\
\hline $\mathrm{E}_{\mathrm{DFB}}$ & & -6171.72063 & -6171.64984 & \\
\hline $\mathrm{E}_{\mathrm{NR}}$ & & -6022.86106 & -6022.80746 & -6022.93169 \\
\hline$\Delta \mathrm{E}_{\mathrm{NR}-\mathrm{DFC}}$ & & 153.27992 & 153.26249 & 153.20936 \\
\hline $\mathbf{E}_{\mathrm{B}}$ & & 4.42035 & 4.42011 & \\
\hline $\mathrm{Xe}$ & 131.30 & $32 s 30 \mathrm{p} 20 \mathrm{~d}^{\mathrm{b}}$ & [19s18p7d] & \\
\hline $\mathrm{E}_{\mathrm{DFC}}$ & & -7446.90026 & -7446.83371 & $-7446.90018^{\circ}$ \\
\hline $\mathrm{E}_{\mathrm{DFB}}$ & & -7441.13047 & -7441.06467 & \\
\hline $\mathrm{E}_{\mathrm{NR}}$ & & -7232.07555 & -7232.05416 & $-7232.13836^{d}$ \\
\hline$\Delta \mathrm{E}_{\mathrm{NR}-\mathrm{DFC}}$ & & 214.82471 & 214.77955 & 214.76182 \\
\hline $\mathrm{E}_{\mathrm{B}}$ & & 5.76979 & 5.76904 & \\
\hline $\mathrm{Hg}$ & 200.59 & $32 \mathrm{~s} 30 \mathrm{p} 20 \mathrm{~d} 14 \mathrm{f}^{\mathrm{b}}$ & {$[19 \mathrm{~s} 19 \mathrm{p} 9 \mathrm{~d} 4 \mathrm{f}]$} & \\
\hline $\mathrm{E}_{\mathrm{DFC}}$ & & -19648.8712 & -19647.8853 & $-19648.8692^{\circ}$ \\
\hline $\mathrm{E}_{\mathrm{DFB}}$ & & -19626.2386 & -19625.2711 & \\
\hline $\mathrm{E}_{\mathrm{NR}}$ & & -18408.5395 & -18408.4158 & $-18408.9915^{\mathrm{e}}$ \\
\hline$\Delta \mathrm{E}_{\mathrm{NR}-\mathrm{DFC}}$ & & 1240.3317 & 1239.4695 & 1239.8777 \\
\hline $\mathrm{E}_{\mathrm{B}}$ & & 22.6326 & 22.6142 & \\
\hline
\end{tabular}

${ }^{\mathrm{a}}$ Universal Gaussian basis set (UGBS) obtained from Ref. [3].

${ }^{b}$ UGBS obtained from Ref. [1].

${ }^{\circ} \mathrm{E}_{\mathrm{DFC}}$ from Ref. [15].

${ }^{d} E_{N R}$ from Ref. [16].

${ }^{\mathrm{e}} \mathrm{E}_{\mathrm{NR}}$ from Ref. [17]. 
Table III. Orbital and total DFC energies for Ge (in hartree)

\begin{tabular}{|c|c|c|c|c|c|c|}
\hline & \multicolumn{2}{|c|}{ Present work } & \multicolumn{2}{|c|}{ Ishikawa et al. } & \multicolumn{2}{|c|}{ Aerts-Nieuwpoort ${ }^{\circ}$} \\
\hline & $32 \mathrm{~s} 30 \mathrm{p} 20 \mathrm{~d}^{\mathrm{a}}$ & [17s14p5d] & $12 \mathrm{~s} 9 \mathrm{p} 5 \mathrm{~d}$ & {$[8 \mathrm{~s} 7 \mathrm{p} 3 \mathrm{~d}]$} & $12 \mathrm{~s} 9 \mathrm{p} 5 \mathrm{~d}$ & {$[8 \mathrm{~s} 7 \mathrm{p} 3 \mathrm{~d}]$} \\
\hline $1 s_{1 / 2}$ & -411.0671 & -411.0651 & -411.0313 & -410.9549 & -411.0314 & -411.2364 \\
\hline $2 s_{1 / 2}$ & -53.45766 & -53.45629 & -53.4338 & -53.4333 & -53.4338 & -53.4545 \\
\hline $3 s_{1 / 2}$ & -7.410432 & -7.408510 & -7.3870 & -7.3818 & -7.3870 & -7.3887 \\
\hline $4 s_{1 / 2}$ & -0.5699943 & -0.5697662 & -0.5432 & -0.5426 & -0.5432 & -0.5434 \\
\hline $2 p_{1 / 2}$ & -47.33620 & -47.33411 & -47.3046 & -47.2829 & -47.3061 & -47.3041 \\
\hline $3 p_{1 / 2}$ & -5.331614 & -5.329534 & -5.3082 & -5.3017 & -5.3018 & -5.3065 \\
\hline $4 p_{1 / 2}$ & -0.2595346 & -0.2597305 & -0.2269 & -0.2265 & -0.2269 & -0.2267 \\
\hline $2 \mathrm{p}_{3 / 2}$ & -46.14768 & -46.14589 & -46.1251 & -46.1141 & -46.1252 & -46.1264 \\
\hline $3 p_{3 / 2}$ & -5.154975 & -5.153066 & -5.1330 & -5.1253 & -5.1330 & -5.1285 \\
\hline $3 \mathrm{~d}_{3 / 2}$ & -1.618571 & -1.616703 & -1.5928 & -1.5867 & -1.5928 & -1.5925 \\
\hline $3 \mathrm{~d}_{5 / 2}$ & -1.591902 & -1.590331 & -1.5662 & -1.5604 & -1.5662 & -1.5662 \\
\hline $\mathrm{E}_{\mathrm{DFC}}$ & -2097.46672 & -2097.46007 & -2097.2333 & -2097.0732 & -2097.232 & -2097.687 \\
\hline $\mathrm{E}_{\mathrm{NR}}$ & -2075.32373 & -2075.31357 & -2075.1628 & -2075.1351 & -2075.195 & -2075.167 \\
\hline$\Delta \mathrm{E}_{\mathrm{NR}-\mathrm{DFC}}$ & 22.14299 & 22.14650 & 22.0705 & 21.9381 & 22.037 & 22.520 \\
\hline
\end{tabular}

${ }^{a}$ Universal gaussian basis set (UGBS) obtained from Ref. [3].

${ }^{\mathrm{b}}$ Energies obtained from Ref. [8].

${ }^{\circ}$ Energies obtained from Ref. [18]. 
Table IV. Orbital and total DFC energies for $\mathrm{Sn}$ (in hartree)

\begin{tabular}{|c|c|c|c|c|c|c|}
\hline & \multicolumn{2}{|c|}{ Present work } & \multicolumn{2}{|c|}{ Ishikawa et al. } & \multicolumn{2}{|c|}{ Aerts-Nieuwpoort ${ }^{\circ}$} \\
\hline & $32 \mathrm{~s} 30 \mathrm{p} 20 \mathrm{~d}^{\mathrm{a}}$ & [19s20p7d] & $15 s 11 p 6 d$ & [10s9p5d] & $15 \mathrm{~s} 11 \mathrm{p} 6 \mathrm{~d}$ & {$[10 \mathrm{~s} 9 \mathrm{p} 5 \mathrm{~d}]$} \\
\hline $1 s_{1 / 2}$ & -1079.318 & -1079.315 & -1079.214 & -1078.068 & -1079.216 & -1083.430 \\
\hline $2 s_{1 / 2}$ & -166.3094 & -166.3057 & -166.2771 & -166.1966 & -166.2778 & -166.8077 \\
\hline $3 s_{1 / 2}$ & -33.55593 & -33.55656 & -33.51711 & -33.50171 & -33.51732 & -33.62210 \\
\hline $4 s_{1 / 2}$ & -5.879111 & -5.881975 & -5.84583 & -5.84297 & -5.84588 & -5.86539 \\
\hline $5 s_{1 / 2}$ & -0.5056921 & -0.5028914 & -0.48298 & -0.48279 & -0.48299 & -0.48458 \\
\hline $2 p_{1 / 2}$ & -154.8782 & -154.8673 & -154.8051 & -154.6618 & -154.7965 & -154.8014 \\
\hline $3 p_{1 / 2}$ & -28.87031 & -28.86903 & -28.82471 & -28.80904 & -28.82291 & -28.82074 \\
\hline $4 p_{1 / 2}$ & -4.246858 & -4.252803 & -4.21375 & -4.21197 & -4.21343 & -4.21177 \\
\hline $5 p_{1 / 2}$ & -0.2436926 & -0.2511663 & -0.21907 & -0.21896 & -0.21906 & -0.21881 \\
\hline $2 \mathrm{p}_{3 / 2}$ & -146.3630 & -146.3584 & -146.3430 & -146.3360 & -146.3438 & -146.2615 \\
\hline $3 p_{3 / 2}$ & -27.26665 & -27.26691 & -27.23275 & -27.23312 & -27.23296 & -27.20170 \\
\hline $4 p_{3 / 2}$ & -3.948922 & -3.956088 & -3.91991 & -3.92012 & -3.91996 & -3.91229 \\
\hline $3 d_{3 / 2}$ & -19.07907 & -19.07708 & -19.02127 & -19.02206 & -19.02117 & -19.01882 \\
\hline $4 d_{3 / 2}$ & -1.329369 & -1.337476 & -1.28785 & -1.28789 & -1.28784 & -1.28656 \\
\hline $3 d_{5 / 2}$ & -18.75280 & -18.75125 & -18.70035 & -18.70199 & -18.70054 & -18.69902 \\
\hline $4 d_{5 / 2}$ & -1.283951 & -1.292503 & -1.24421 & -1.24446 & -1.24424 & -1.24319 \\
\hline $\mathrm{E}_{\mathrm{DFC}}$ & -6176.14098 & -6176.06995 & -6175.2283 & -6172.5075 & -6175.204 & -6184.710 \\
\hline $\mathrm{E}_{\mathrm{NR}}$ & -6022.86106 & -6022.80746 & -6022.4239 & -6022.0819 & -6022.429 & -6022.087 \\
\hline$\Delta \mathrm{E}_{\mathrm{NR}^{-} \mathrm{DFC}}$ & 153.27992 & 153.26249 & 152.8044 & 150.4256 & 152.775 & 162.623 \\
\hline
\end{tabular}

${ }^{a}$ Universal gaussian basis set (UGBS) obtained from Ref. [3].

${ }^{b}$ Energies obtained from Ref. [19].

${ }^{c}$ Energies obtained from Ref. [18]. 


\section{References}

[1] F. E. Jorge and A. B. F. da Silva, J. Chem. Phys. 104, 6278 (1996).

[2] F. E. Jorge and A. B. F. da Silva, J. Chem. Phys. 105, 5503 (1996)

[3] F. E. Jorge and A. B. F. da Silva, J. Mol Struct. Theochem 394, 101 (1997).

[4] F. E. Jorge, T. B. Bobbio, and A. da Silva, Chem. Phys. Lett. 263, 775 (1996).

[5] F. E. Jorge, M. T. Barreto and A. B. F. da Silva, Chem. Phys. 221, 45 (1997).

[6] O. Matsuoka and S. Huzinaga, Chem. Phys. Lett. 140, 567 (1987).

[7] O. Matsuoka, Chem. Phys. Lett. 195, 184 (1992).

[8] Y. Ishikawa, H. Sekino and R. C. Binning Jr., Chem. Phys. Lett. 165, 237 (1990).

[9] Y. Ishikawa, Chem. Phys. Lett. 179, 291 (1991).

[10] Y. Ishikawa, Int. J. Quantum Chem. Symp. 25, 113 (1991).

[11] L. Visscher, P. J. C. Aerts, O. Visser and W. C. Nieuwpoort, Int. J. Quantum Chem. Symp. 25, 131 (1991).
[12] F. E. Jorge and A. B. F. da Silva, Chem. Phys. Lett. 289, 469 ( ] 998).

[13] Y. Ishikawa and H. M. Quiney, Int. J. Quantum Chem. Symp. 21, 523 (1987)

[14] R. E. Stanton and S. Havriliak, J. Chem. Phys. 81, 1910 (1984).

[15] I. P. Grant, B. J. McKenzie, P. H. Norrington, D. F. Mayers and N. C. Pyper, Comput. Phys. Commun. 21, 207 (1980).

[16] C. F. Bunge, J. A. Barrientos, A. V. Bunge and J. A. Cogordan, Phys. Rev. A 46, 3691 (1992).

[17] T. Koga, S. Watanabe and A. J. Thakkar, Int. J. Quantum Chem. 54, 261 (1995).

[18] P. J. C. Aerts and W. C. Nieuwpoort, Int. J. Quantum Chem. 19, 267 (1986); P. J. C. Aerts, Towards Relativistic Quantum Chemistry, Thesis, University of Groningen (1986).

[19] Y. Ishikawa, Chem. Phys. Lett. 212, 547 (1993).

[20] Y. Ishikawa and H. Sekino, Chem. Phys. Lett. 165, 243 (1990). 